\title{
Renal Pelvis Fibroepithelial Polyp
}

National Cancer Institute

\section{Source}

National Cancer Institute. Renal Pelvis Fibroepithelial Polyp. NCI Thesaurus. Code C6182.

A benign, mesodermal tumor located in the renal pelvis. 